\title{
Cognitive Deficits in Chronic Heart Failure
}

\author{
Susan J. Pressler, DNS, RN [Professor], \\ School of Nursing University of Michigan Ann Arbor, Michigan \\ Usha Subramanian, MD, MS [Associate Professor, Physician], \\ Department of Medicine Indiana University Indianapolis, Indiana and Richard L. Roudebush \\ Veteran Affairs Medical Center Indianapolis, Indiana
}

David Kareken, PhD [Associate Professor],

Divisions of Neuropsychology Indiana University Indianapolis, Indiana

Susan M. Perkins, PhD [Associate Professor],

Biostatistics Indiana University Indianapolis, Indiana

Irmina Gradus-Pizlo, MD [Associate Professor],

Krannert Institute of Cardiology Indiana University Indianapolis, Indiana

Mary Jane Sauve, DNSc, RN [Adjunct Professor],

School of Nursing University of California at San Francisco San Francisco, California

Yan Ding, MS [Biostatistician],

Biostatistics University of California at San Francisco San Francisco, California

JinShil Kim, PhD, RN [Assistant Professor],

College of Nursing Michigan State University East Lansing, Michigan

Rebecca Sloan, PhD, RN [Associate Professor],

School of Nursing Indiana University Indianapolis, Indiana

Heather Jaynes, MSN, RN [Clinical Nurse Specialist], and

School of Nursing Indiana University Indianapolis, Indiana

Rose Mary Shaw, PsyD [Clinical Psychologist]

Wright State University Dayton, Ohio

\section{Abstract}

Background: Patients with heart failure (HF) have been found to have cognitive deficits, but it remains unclear whether these deficits are associated with $\mathrm{HF}$ or with aging or comorbid conditions common in HF.

Objectives: To 1) determine the types, frequency, and severity of cognitive deficits among patients with chronic HF compared to age- and education-matched healthy participants and participants with major medical conditions other than HF; and 2) evaluate the relationships between HF severity, age, and comorbidities and cognitive deficits.

Correspondence: Susan J. Pressler, RN, DNS Professor University of Michigan School of Nursing 400 N. Ingalls, Ann Arbor, MI 48109 Phone: (734) 763-5650 Fax: (734) 936-5525 spressle@umich.edu.

This is a PDF file of an unedited manuscript that has been accepted for publication. As a service to our customers we are providing this early version of the manuscript. The manuscript will undergo copyediting, typesetting, and review of the resulting proof before it is published in its final citable form. Please note that during the production process errors may be discovered which could affect the content, and all legal disclaimers that apply to the journal pertain. 
Methods: A sample of 414 participants completed the study (249 HF patients, 63 healthy and 102 medical participants). The HF patients completed measures of HF severity, comorbidity (multiple comorbidity, hypertension, depressive symptoms), and neuropsychological functioning. Blood pressure and oxygen saturation were assessed at interview; clinical variables were abstracted from records. Participants in the comparison groups completed the same measures as the HF patients except those specific to HF.

Results: Compared to the healthy and medical participants, HF patients had poorer memory, psychomotor speed, and executive function. Significantly more HF patients (24\%) had deficits in three or more domains. Higher (worse) HF severity was associated with more cognitive deficits; HF severity interacted with age to explain deficits in executive function. Surprisingly, men with HF had poorer memory, psychomotor speed, and visuospatial recall ability than women. Multiple comorbidity, hypertension, depressive symptoms, and medications were not associated with cognitive deficits in this sample.

Discussion: Heart failure results in losses in memory, psychomotor speed, and executive function in almost one fourth of patients. Patients with more severe HF are at risk for cognitive deficits. Older patients with more severe HF may have more problems in executive function and men with HF may be at increased risk for cognitive deficits. Studies are urgently needed to identify the mechanisms for the cognitive deficits in HF and test innovative interventions to prevent cognitive loss and decline.

\section{Keywords}

heart failure; cognitive deficits; cognitive impairment

Heart failure (HF) remains a major public health problem, affecting more than 5.7 million Americans (Lloyd-Jones et al., 2009). The estimated direct and indirect costs of HF care in the United States in 2009 are estimated to be 37.2 billion dollars. Although the 12-month mortality rates for $\mathrm{HF}$ are high, 80 to $85 \%$ of patients survive longer than 12 months. Health-related quality of life is diminished by dyspnea, fatigue, and depressive symptoms (Bennett et al., 2003).

Patients have reported that cognitive deficits are another problem that diminishes their health-related quality of life (Bennett, Cordes, Westmoreland, Castro, \& Donnelly, 2000). These deficits may compromise patients' reasoning and decision-making abilities, thereby limiting their ability to perform self-care. Among 1,583 elderly patients hospitalized with $\mathrm{HF}$, cognitive impairment was associated with significantly higher mortality (Zuccala et al., 2003). Cognitive deficits in HF have been investigated, but the studies have yielded mixed results about the association between HF and cognitive deficits (Bennett \& Sauve, 2003; Bennett, Sauve, \& Shaw, 2005; Pressler, 2008; Pullicino et al., 2008; Vogels, Scheltens, Schroeder-Tanka, \& Weinstein, 2007). Some investigators have found that HF and HF severity were associated with cognitive deficits, but others have not found significant associations between $\mathrm{HF}$ and cognitive deficits. Limitations of past studies may explain the equivocal results. First, investigators used global screening questionnaires to assess cognitive deficits. Screening questionnaires are clinically feasible but lack the sensitivity needed to detect subtle cognitive deficits and thoroughly evaluate the association between HF and types of cognitive deficits. Second, investigators conducted studies among small or select samples (e.g., transplant candidates) with few women and minority participants. Finally, investigators often did not include comparison groups or variables of age and comorbid conditions; these are needed to fully evaluate HF patients' cognitive deficits.

The conceptual framework guiding the study is presented in detail elsewhere (Bennett et al., 2005). Deficits in the cognitive domains of language, working memory, memory, 
visuospatial ability, psychomotor speed, and executive function are hypothesized to occur in HF patients. More severe HF is proposed to be associated with more cognitive deficits. The mechanism for cognitive deficits in HF remains undetermined, but the most likely etiology is decreased cardiac output leading to inadequate cerebral perfusion and oxygenation (Vogels, Oosterman et al., 2007; Woo, Macey, Fonarow, Hamilton, \& Harper, 2003). The brain, particularly the highly plastic areas, requires large amounts of oxygen to function adequately. As such, the hippocampus, amygdala, frontal lobes, and cerebellum are vulnerable to oxygen deprivation and may be selectively damaged during hypoxic episodes (Rains, 2002). Hippocampal damage is important because of its role in the acquisition of new information and generation of memory, which are essential for performing activities of daily living and HF self-care.

The brain changes associated with sudden or abrupt reductions in cerebral blood flow have been well characterized, but less is known about the effects of chronic reductions in cerebral blood flow (Vogels, Oosterman et al., 2007; Zipes, Libby, Bonow, \& Braunwald, 2005). In an animal model, transient cerebral ischemia significantly decreased cells in the CA1 hippocampal region; longer periods of ischemia (5 and 10 minutes) produced increased cell loss and were associated with deficits in spatial learning (Briones \& Therrien, 2000). In HF patients, structural brain changes consistent with decreased cerebral perfusion and oxygenation have been identified, including loss of gray matter volume in the frontal cortex and parahippocampal areas (Woo et al., 2003) and silent ischemic strokes (Siachos et al., 2005).

Two recent investigations using magnetic resonance imaging technologies found brain structural changes among HF patients. Kumar and colleagues (Kumar et al., 2009) documented significant decreases in the volumes of the right and left mammillary bodies and the cross-sectional areas of the fornix fibers of HF patients $(n=17)$ compared to agematched healthy participants $(n=50)$. Kumar's study provides a structural basis for memory deficits in HF because the mammillary bodies and fornix fibers are linked to severe anterograde and spatial memory deficits in animal and human studies (Kumar et al., 2009). Woo and colleagues (Woo, Kumar, Macey, Fonarow, \& Harper, 2009) identified injured areas in the hippocampus, the hippocampal output fibers that project to the septum and the anterior thalamus, the caudate nuclei, and the anterior midportions of the corpus callosum of 13 HF patients (mean age 54.6 years) when compared to 49 healthy control participants (mean age 50.6 years). All brain sites of the HF patients showed significantly more differences suggestive of brain damage compared to the control participants. The types of injuries identified by Woo and colleagues involve structures that support short-term memory, learning, and executive function.

However, variables of age, multiple comorbid conditions, and two specific comorbid conditions common in HF (i.e., hypertension and depressive symptoms) are alternative explanations for cognitive deficits in HF. In the present study, these factors were evaluated as variables that contribute to cognitive deficits in HF. Age-related cognitive decline occurs in some individuals and the incidence of HF increases with age. Multiple comorbid conditions are present because HF patients are older. Hypertension is a common condition in $\mathrm{HF}$ that is associated with cognitive deficits (Waldstein, 2003). Depression is common in $\mathrm{HF}$, occurring in $21.5 \%$ of patients in a meta-analysis of 36 studies (Rutledge, Reis, Linke, Greenberg, \& Mills, 2006). Depressive symptoms overlap with signs of cognitive deficits (e.g., decreased attention). In a population-based study, comorbidity (i.e., depression and anemia) attenuated cognitive impairment in HF (Pullicino et al., 2008). However, cognitive impairment was measured during a telephone interview using a brief questionnaire rather than a neuropsychological test battery. 
Educational level, gender, and medications were evaluated as covariates in the current study because they may contribute to cognitive deficits noted in HF. Educational level was evaluated as a covariate because it is associated with neuropsychological test scores. Older women who received hormone replacement therapy were found to have an increased incidence of Alzheimer disease, although recent data have called this finding into question (Shumaker et al., 2003). Few studies have had sufficient numbers of women with HF to examine gender differences in cognitive deficits. In a retrospective study of patients awaiting cardiac transplant, women $(n=158)$ had significantly greater deficits in language and psychomotor speed than men $(n=602)$, but men had more deficits in verbal learning (Putzke et al., 1997). Medications with a known influence on cognition were included as a covariate because of their potential to contribute to cognitive deficits. No studies were found that evaluated medications in relationship to cognitive deficits in HF.

To address limitations of past studies and more fully evaluate cognitive deficits in HF, patients with a spectrum of HF severity were studied and their cognitive function was compared to the function of two samples: healthy individuals and individuals with major chronic medical conditions. The first aim was to determine the types, frequency, and severity of cognitive deficits (in domains of language, working memory, memory, visuospatial ability, psychomotor speed, and executive function) among patients with chronic HF. The second aim was to evaluate the relationships between HF severity, age, and medical comorbidities and cognitive deficits. We hypothesized that: 1) HF patients have significantly more cognitive deficits than age- and education-matched comparison participants; and 2) increased HF severity, age, and comorbidity are associated with more cognitive deficits in HF.

\section{Methods}

Procedures

This study used a comparative design to evaluate cognitive deficits among the three groups and an explanatory correlational design to evaluate the relationships between HF severity, age, and comorbidity and cognitive deficits among the HF patients (Bennett et al., 2005). Data were collected from September 2004 to May 2008. The HF patients were recruited by clinic staff from one general medicine practice and six HF clinics in a Midwestern metropolitan city. Potentially eligible participants were approached by clinic staff, and if interested in the study, their names and contact information were provided to members of the research team. Eighty patients declined participation for the following reasons: lack of interest $(n=43)$; lack of time $(n=12)$; too sick $(n=9)$; unknown $(n=5)$; family issues $(n=$ $2)$; did not remember being approached about study $(\mathrm{n}=1)$; moving out of state $(\mathrm{n}=1)$; had too many doctors' appointments $(\mathrm{n}=1)$; wanted to discuss with family $(\mathrm{n}=1)$; wanted to take consent form home and be approached at another appointment $(\mathrm{n}=1)$; and other $(\mathrm{n}=$ 4). The participants in the healthy and medical groups were recruited from one general medicine practice, family members of HF patients, and volunteers who responded to advertisements. The study was approved by the institutional review boards at the sites. All participants provided written informed consent prior to data collection.

Eligible participants completed face-to-face interviews at a location preferred by them (i.e., clinics where the patients received care, patients' homes, or the school of nursing). Two research team members completed each interview; one was responsible for collection of the data for sociodemographics, HF severity, and comorbidities and the second for collection of the neuropsychological data. The senior neuropsychologist investigator trained the testers according to guidelines for neuropsychological test administration and scoring procedures. Comparison group participants provided all data except HF-related variables. The length of time to obtain consent and administer the questionnaires and tests was 90 to 120 minutes. 
All participants were asked to identify a family member who could provide an assessment of their cognitive status (Hachinski et al., 2006). Family members were interviewed by telephone using a structured questionnaire.

\section{Sample}

Eligibility criteria for the HF patients included a diagnosis of chronic HF documented by echocardiography, nuclear imaging, or cardiac catheterization within the past two years with findings of systolic $\mathrm{HF}$ and left ventricular ejection fraction less than or equal to $40 \%$ (Zipes et al., 2005). Eligibility criteria for the healthy participants included living independently and/or able to care for self and absence of any major medical conditions. Participants who had controlled cardiovascular risk factors (i.e., mild hypertension with blood pressure less than 140/90 or mild hyperlipidemia with total cholesterol less than $200 \mathrm{mg} / \mathrm{dL}$ ) were eligible for the healthy group. Eligibility criteria for the medical participants included a current medical diagnosis of a major chronic disorder other than HF (e.g., diabetes, uncontrolled hypertension). Categorization of participants as healthy or medical group members was verified by two of the investigators. Persons were excluded from participation in all groups if they had conditions known to cause cognitive deficits (e.g., history of or current major alcohol or substance abuse, history of major psychiatric or neurological disorders, encephalopathy, renal failure requiring dialysis) or terminal illness.

\section{Measures}

Heart failure severity-Heart failure severity was measured by the New York Heart Association (NYHA) Class (New York Heart Association Criteria Committee, 1964) during the interview. Although other more invasive measures provide detailed information about HF severity, the NYHA Class was used as a measure of HF severity in this study because it is clinically feasible with low respondent burden and has documented validity and reliability in persons with cardiac disorders (Bennett, Riegel, Bittner, \& Nichols, 2002). Oxygen saturation, assessed at the interview by pulse oximetry, was used as a severity indicator that would detect severe resting decompensation.

Comorbidity-The Charlson Comorbidity Index was used to measure multiple comorbidity (Charlson, Pompei, Ales, \& MacKenzie, 1987). In this Index, participants are asked whether they have a history of medical problems in 12 areas with responses of "yes" or "no". The medical problems reported are weighted by severity with each rating being worth 1, 3, or 6 points. A summary score is obtained; higher scores represent more and/or more severe comorbid conditions. In other populations, the comorbidity categories of the Index predicted 1-year mortality and health care costs (Perkins et al., 2004).

Blood pressure-Blood pressure measurement was assessed during the interview. Hypertension was defined as blood pressure higher than 140/90 $\mathrm{mm} \mathrm{Hg}$ for patients without diabetes mellitus and 130/80 $\mathrm{mm} \mathrm{Hg}$ for patients with diabetes mellitus (National Heart, Lung, and Blood Institute, 2003).

Depressive symptoms-Depressive symptoms were measured using the Patient Health Questionnaire-8 (PHQ-8) (Kroenke \& Spitzer, 2002). The PHQ-8 consists of the first 8 items of the PHQ-9, which was developed based on the 9 depression criteria of the Diagnostic and Statistical Manual of Mental Disorders, $4^{\text {th }}$ edition. Item 9 requires that participants respond to whether they have had suicidal ideation; this item is not required when assessing nonpsychiatric populations (Kroenke \& Spitzer, 2002). Psychometric properties of the PHQ-8 are similar to the properties of the PHQ-9. On the PHQ-8, possible scores range from 0 to 24 . Scores of 1 to 4 indicate no depressive symptoms; 5 to 9 mild symptoms; 10 to 14 moderate symptoms, 15 to 19 moderately severe symptoms; and 20 to 
24 severe depressive symptoms. Scores of 10 or higher indicate the need for possible treatment. Validity and reliability have been documented (Kroenke \& Spitzer, 2002). Reliability estimates of the PHQ-9 were .89 and .86 among 3000 primary care and 3000 obstetrical and gynecological patients, respectively (Kroenke, Spitzer, Williams, 2001). The Cronbach's alpha in the current sample was .82.

Covariates-Educational level and gender were evaluated as covariates. Medications with known effects on cognitive function were evaluated for their relative contribution to cognitive deficits. Medications included were those with a primary central nervous system indication plus those with prominent central nervous system adverse effects: (a) major tranquilizers or anti-psychotic medications; (b) anti-depressants including lithium; (c) Alzheimer disease medications (cholinesterase inhibitors); (d) atropine and scopolamine; (e) steroid hormones including estrogens; (f) beta-adrenergic antagonists (especially propranolol); (g) narcotic analgesics; (h) anti-Parkinson's medications (e.g., L-dopa); (i) anti-epileptic medications (phenytoin; gabapentin); and (j) barbiturates (Hardman \& Limbird, 2001). The medications were analyzed as dichotomous covariates and patients who were prescribed one or more of the medications from the above groups were considered as receiving a medication known to alter cognitive function while patients not prescribed the above medications were considered as not receiving a medication known to alter cognition.

Cognitive deficits-The neuropsychological test battery administered to all participants was designed to measure the cognitive domains most likely to be impaired in vascular cognitive disorders and commonly sampled in a neuropsychological examination for these disorders (Hachinski et al., 2006). The battery required approximately 60 minutes to administer. The tests have documented validity and reliability, including the ability to discriminate between persons with normal cognitive functioning, mild cognitive impairment, and dementia. Table 1 presents the cognitive domains evaluated, the neuropsychological tests administered, and brief descriptions of the tests.

Participants' family members completed a 24-item family interview questionnaire with responses of "yes" and "no" to support validation of the neuropsychological tests. This questionnaire measured family members' observations about whether their participants had changes in cognition or behavior in the past several years. It yields four subscale scores in memory difficulty ( 8 items), information recall ( 5 items), judgment (4 items), and behavior (7 items). Higher scores indicate more cognitive and/or behavioral problems.

\section{Statistical analysis}

Descriptive statistics were computed for study variables and compared among the HF, healthy, and medical participant groups using ANOVA, Chi-square, or Fisher' Exact tests. To achieve aim one, ANCOVA was first used to compare the raw scores of the neuropsychological tests among the three groups, adjusting for age, premorbid intellect, and education. Next, Z-scores of the neuropsychological tests were constructed and compared among the groups because local normative samples may better calibrate levels of deficits than published norms. For the healthy participant group, each test measure was regressed on age, premorbid intellect, and education. Recall of geometric figures (Figure Copy) and the Trail Making Test Part A scores were natural log transformed to achieve normality in the residuals. Predicted values for the HF, healthy, and medical participants were obtained from these regression models and Z-scores calculated. To compare the Z-scores between the HF patients and the healthy and medical participants, t-tests (equal or unequal variance as appropriate) were used for cognitive domains measured by a single neuropsychological test score (global cognitive function and language), and Hotelling's $\mathrm{T}^{2}$ tests for domains measured by two or more test scores (working memory, memory, visuospatial ability, 
psychomotor speed, and executive function). Tests were assessed at the .05 level of significance for single score domains and by Bonferroni correction for multiple score domains when the Hotelling's $\mathrm{T}^{2}$ test was significant at the .05 level.

Cognitive impairment cutoff scores were defined from the healthy participants' Z-scores using the $7^{\text {th }}$ percentile. Patients who performed at or below the $7^{\text {th }}$ percentile cutoff were regarded as being cognitively impaired in that domain (Peterson et al., 1995). Chi-square or Fisher's Exact test as appropriate were used to compare the percent of HF patients with cognitive impairment to the percent of healthy and medical participants with cognitive impairment, using Bonferroni corrections for multiple measures. Family-reported changes in cognition were compared using Kruskal-Wallis for overall differences and Wilcoxon-Rank sum tests for pairwise comparisons.

To accomplish aim two, a series of multiple linear regression analyses were conducted using the data from the HF patients. Each neuropsychological variable was entered as the dependent variable in separate regression equations. Independent variables were NYHA Class, oxygen saturation, age, and comorbidity (Charlson Comorbidity Index, hypertension, and depressive symptoms); interactions between NYHA Class and age and comorbidity were examined at a more stringent .01 significance level to control for multiple testing. Covariates were premorbid intellect, education, gender, and medications with an influence on cognitive function.

\section{Results}

A total of 414 participants completed the study, including 249 HF patients, 63 healthy participants, and 102 medical participants. Sample characteristics are presented in Table 2. The demographic characteristics were balanced among the groups with the exceptions that the healthy participants were younger than the HF and medical participants and the HF group had more men and more were married. Some participants were unable to complete the full neuropsychological test battery due to sensory or health problems; sample size varies slightly across analyses.

Comparisons among the groups of oxygen saturation, comorbidity, blood pressure, and depressive symptoms are presented in Table 3. Compared to participants in the other two groups, the HF patients had significantly more or more severe comorbid conditions, more or more severe depressive symptoms, and lower diastolic blood pressure. Compared to the medical participants, the HF patients had significantly lower systolic blood pressure.

The neuropsychological test scores of the HF patients were compared to the healthy and medical participants. Raw score comparisons are presented in Table 3. The HF patients had worse scores than the healthy and medical participants in total recall memory and than the medical participants in delayed recall memory. The HF patients had slower psychomotor speed than the healthy and medical participants. The HF patients had worse scores in executive function.

The Z-score means and standard deviations of the neuropsychological test results among the groups are presented in Table 4. Compared to the medical participants, the HF patients had poorer performance in memory, slower psychomotor speed, and poorer executive function.

Using the $7^{\text {th }}$ percentile Z-score as a cutoff, significantly more HF patients had memory impairment (total recall) compared to healthy and medical participants (Table 5). Among the groups, $23 \%$ of the HF patients had impaired total recall memory compared to $8 \%$ of the healthy and $9 \%$ of the medical participants. In psychomotor speed, $19 \%$ of the HF patients were impaired compared to $8 \%$ and $6 \%$ of the healthy and medical participants, respectively. 
On executive function measures, $19 \%$ and $16 \%$ of the HF patients performed as impaired, compared to $8 \%$ of the healthy and $10 \%$ and $7 \%$ of the medical participants. Twenty-four percent of the HF patients were impaired in three or more cognitive domains, compared to $14 \%$ of the healthy and $12 \%$ of the medical participants. Post hoc analysis indicated HF patients had more impairment than medical participants.

Family members of the HF patients $(n=230)$ reported that the patients had more cognitive changes than family members of healthy $(n=53)$ and medical $(n=89)$ participants. Differences were found in memory difficulty (Chi-square $=22.03, \mathrm{p}<.0001$ ), information recall (Chi-square $=12.27, \mathrm{p}=.002)$, judgment (Chi-square $=7.06, \mathrm{p}=.029)$, and behavior (Chi-square $=15.43, \mathrm{p}=.0004)$. In post hoc analyses using Wilcoxon-Rank sum with Bonferroni adjustment to alpha $=.0167$, more changes were reported by family members of HF patients than family members of healthy and medical participants in memory difficulty $(\mathrm{p}<.0001)$ and than healthy participants in information recall $(\mathrm{p}=.002)$ and behavior $(\mathrm{p}=$. 0004).

In the regression models, HF severity (NYHA Class) was significantly associated with memory (Hopkins total recall), visuospatial ability (Figure Memory recall), psychomotor speed (Digit Symbol), and executive function (Trail Making Test Part B and Controlled Oral Word Association). Patients with NYHA Class I and II HF had higher Z-scores overall, indicating better cognitive function. Heart failure severity (NYHA Class) and age significantly interacted to explain executive function (Trail Making Test B and Controlled Oral Word Association) (Tables 6 and 7). Older age was associated with better executive function scores (Trail Making Test B) for NYHA Class II and with worse executive function scores (Controlled Oral Word Association) for NYHA Class IV.

In the regression models, men had significantly poorer total and delayed recall memory $(\mathrm{t}=$ 4.7 and 25.2, respectively, $\mathrm{p}<.0001$ in both cases), psychomotor speed (Digit Symbol; $\mathrm{t}=$ $12.2, \mathrm{p}=.0006$ ), and visuospatial ability (Figure Memory recall; $\mathrm{t}=2.0, \mathrm{p}=.046$ ) than women. There were no significant mean differences between men and women in age, premorbid intellect, education, LVEF, oxygen saturation, multiple comorbidity, or depressive symptoms. Women were more likely to have NYHA Class III and IV than men $(59 \%$ vs. $46 \%$, Chi-square $=4.38, \mathrm{p}=.04)$.

Multiple comorbidity, depressive symptoms, and hypertension were not significant explanatory variables for cognitive deficits. Medications with a known influence on cognitive function were entered into the equations but were not significant explanatory variables for cognitive deficits.

\section{Discussion}

Three important results emerged from this study. First, the $249 \mathrm{HF}$ patients had poorer memory, psychomotor speed, and executive function than the 63 healthy and 102 medical participants and $24 \%$ of the HF patients had cognitive deficits in three or more domains. Second, patients with more severe HF had poorer total recall memory, visuospatial recall ability, psychomotor speed, and executive functioning. Older patients with more severe HF had poorer executive functioning. Third, men with HF had poorer memory, psychomotor speed, and visuospatial recall ability than women.

Consistent with past studies, HF patients had poorer performance compared to healthy and medical participants in memory, psychomotor speed, and executive functioning (Bennett \& Sauve, 2003; Vogels, Scheltens et al., 2007; Pressler, 2008; Vogels, Oosterman et al., 2007). Some inconsistencies remain about the relationship between HF severity and cognitive deficits. In the current study, more severe HF was associated with poorer total recall 
memory, poorer visuospatial recall ability, psychomotor slowing, and poorer executive function, although not across all tests. Heart failure severity (NYHA classes II and IV) and age interacted to explain executive function. It is possible that the trajectory of chronic HF, with episodic decompensations interspersed with periods of stability, contributes to the varying extent of deficits in individual patients. The multiple anatomic defects in the brain described by Kumar et al. (2009) and Woo et al. (2009) lend credence to cerebral events being part of the constellation of systemic damage in chronic HF.

No significant differences were found in the cognitive domains of language, working memory, and visuospatial ability among the HF patients when compared to the healthy and medical participants. It is possible that the measures used to assess these domains were not sensitive or challenging enough to detect differences among the groups given that the groups were matched on education and premorbid intellect. However, the measures (Boston Naming Test, Digit Span, and Figure Copy and Memory) have discriminated between healthy and cognitively impaired individuals in other studies and are recommended for use when evaluating vascular cognitive disorders (Hachinski et al., 2006). An attempt was made to minimize environmental distractions during testing, which may have facilitated the attention of patients. Although not tested in this study, it is possible that the brain structures responsible for language and working memory are less sensitive to cerebral hypoperfusion than the areas responsible for memory, psychomotor speed, and executive function.

The ability to detect the relationship between HF severity and cognitive deficits may have been limited by measurement factors. The NYHA Class is valid, reliable, and clinically feasible, but more invasive methods are required to fully evaluate HF severity. Selection bias may have prevented demonstrating a systematic relationship between HF severity and cognitive deficits across all tests because only those NYHA Class IV patients who were well enough completed the interviews; others were too sick to participate.

Multiple comorbidity, hypertension, and depressive symptoms were not associated with the cognitive deficits in this sample of HF patients. These results are in contrast to a study that reported that depression and anemia predicted cognitive impairment in HF (Pullicino et al., 2008). Participants in that study were categorized as probably having HF based on symptoms of dyspnea and orthopnea and cognitive impairment and depression were measured using 6- and 4-item questionnaires, respectively, that were administered by telephone interviews. These differences between the two studies in the validation of the HF diagnosis, measures for cognitive deficits, and administration methods, may explain the differences in the results. In the current study, patients had a definitive HF diagnosis and cognitive deficits were measured during face-to-face interviews using valid objective neuropsychological tests. Depressive symptoms were measured as a subjective phenomenon using the valid and reliable PHQ-8 that is sensitive to depression.

The finding that men with HF had poorer memory, psychomotor speed, and visuospatial recall ability than women with HF was unexpected. This was the first controlled study found to report this result. Interestingly, there were no differences between men and women in age, premorbid intellect, education, LVEF, oxygen saturation, multiple comorbidity, or depressive symptoms. The HF group had $63 \%$ men and $37 \%$ women; the higher percentage of men in the sample may have contributed to the finding of more cognitive deficits in men than women. In one of the few studies reporting gender differences, Putzke (Putzke et al., 1997) did find that men had more deficits in verbal learning. It is intriguing to speculate that sex hormones may play a role in the fewer deficits of women but other factors such as duration of HF, thromboembolic risk, incidence of diabetes, smoking, and concomitant medications may also have been contributory to the deficits. One potential factor, severity of $\mathrm{HF}$, does not seem as likely of an explanation of the gender differences since NYHA Class 
III and IV HF was more prevalent in women yet men had more profound cognitive deficits. The type and extent of cognitive deficits related to gender need to be confirmed in an adequately powered prospective study that controls for demographic, biological, clinical, and social variables that may differ between men and women.

Strengths of the current study include a carefully characterized sample of HF patients with representative numbers of women and minority (African-American) participants; comparison groups matched on premorbid intellect and education; use of valid objective neuropsychological tests to measure cognitive deficits; and evaluation of comorbid conditions that could contribute to cognitive deficits. The HF patients were recruited from seven sites; this increases the representative of the sample. A limitation of the study was that the healthy participants were younger than the HF patients and this difference cannot be excluded as a reason for the differences observed in cognitive deficits. Despite efforts to enroll age-matched healthy elders without major medical conditions, most participants over age $60 \mathrm{had}$ at least one medical condition. This is expected; 74\% of Americans age 65 years and older have one or more chronic health conditions (National Institute on Aging, 2007). The finding that the HF patients had more deficits than the medical participants who were age-matched lends support to HF as the cause of the deficits. The results are not generalizable to the entire HF population because only patients with documented systolic dysfunction and without obvious reasons for cognitive deficits were included.

In conclusion, cognitive deficits are a common problem that affects nearly one fourth of patients with chronic systolic HF. Memory deficits are most common, followed by psychomotor slowing and decreased executive function. Patients with more severe HF had more cognitive deficits, and older patients with more severe HF had more deficits in executive function. Men with HF had poorer memory, psychomotor speed, and visuospatial recall ability than women. Future studies are needed to determine the etiology of the cognitive deficits in order to prevent them and to test novel interventions targeted at preventing and delaying cognitive loss and decline.

The finding that many patients with HF have cognitive deficits has important clinical implications. Patients need to be carefully evaluated for cognitive deficits and whether these deficits alter their ability to perform activities of daily living and comply with HF self-care behaviors. Older patients with more severe HF may be most at risk for deficits and diminished decision-making abilities. These patients require assistance from family caregivers and would likely benefit from disease management and transitional care programs.

\section{Acknowledgments}

This study was funded by the National Institute of Nursing Research (R01NR008147).

Thank you for assistance with recruitment and data collection to Kari Berron, RN, MSN and Mary Walsh, MD, The Care Group, Indianapolis, IN; Joan Barr, RN, MSN, James Hafer, RN, MSN, Clarian Health Partners, Indianapolis, IN; Cynthia Adams, RN, PhD, Sara Fickle, RN, MSN, Cynthia Kennedy, RN, MSN, Jeanne Majors, RN, MSN, Sharon Sipos, AD, Linda Trowbridge, RN, Community Health Network of Indianapolis; Maureen Bender, RN, MSN, Mandrelle Dreu Doerstler, RN, MSN, Amber Hunt, BA, Janet Kain, MS, Klarisa Laye, RN, BSN, Elisa Liou, BA, Kristen Mather, RN, MSN, Laura Parker, Janice Powers, RN, PhD, Indiana University School of Nursing.

\section{References}

Bennett JA, Riegel B, Bittner V, Nichols J. Validity and reliability of the NYHA classes for measuring research outcomes in patients with cardiac disease. Heart \& Lung 2002;31:262-270. [PubMed: 12122390] 
Bennett SJ, Cordes DK, Westmoreland G, Castro R, Donnelly E. Self-care strategies for symptom management in patients with heart failure. Nursing Research 2000;49:139-145. [PubMed: 10882318]

Bennett SJ, Oldridge NB, Eckert G, Embree JL, Browning S, Hou N, et al. Comparison of quality of life measures in heart failure. Nursing Research 2003;52:207-216. [PubMed: 12867777]

Bennett SJ, Sauve MJ. Cognitive deficits in patients with heart failure: a review of the literature. Journal of Cardiovascular Nursing 2003;18:219-242. [PubMed: 12837012]

Bennett SJ, Sauve MJ, Shaw RM. A conceptual model of cognitive deficits in chronic heart failure. Journal of Nursing Scholarship 2005;37:222-228. [PubMed: 16235862]

Benton, AL.; Hamsher, K deS. Multilingual Aphasia Exam Manual. University of Iowa; Iowa City, IA: 1978 .

Brandt, J.; Benedict, RHB. Hopkins Verbal Learning Test - Revised. Psychological Assessment Resources; Lutz, FL: 2001.

Briones TL, Therrien B. Behavioral effects of transient cerebral ischemia. Biological Research 2000;1:276-286.

Charlson ME, Pompei P, Ales KL, MacKenzie CR. A new method of classifying prognostic comorbidity in longitudinal studies: development and validation. Journal of Chronic Disease 1987;40:373-383.

Folstein MF, Folstein SE, McHugh PR. Mini-mental state: a practical method for grading the cognitive state of patients for the clinician. Journal of Psychiatric Research 1975;12:189-198. [PubMed: 1202204]

Hachinski V, Iadecola C, Petersen RC, Breteler MM, Nyenhuis DL, Black SE, et al. National Institute of Neurological Disorders and Stroke-Canadian Stroke Network Vascular Cognitive Impairment Harmonization Standards. Stroke 2006;37:2220-2241. [PubMed: 16917086]

Hardman, JG.; Limbird, LE., editors. Goodman and Gilman's the Pharmacological Basis of Therapeutics. 10th ed.. McGraw-Hill; NY: 2001.

Kaplan, EF.; Goodglass, H.; Weintraub, S. The Boston Naming Test. 2nd ed.. PRO-ED Inc; Austin, TX: 2001.

Kroenke K, Spitzer RL. The PHQ-9: a new depression diagnostic and severity measure. Psychiatric Annals 2002;32:1-7.

Kroenke K, Spitzer RL, Williams JBW. The PHQ-9: validity of a brief depression severity measure. Journal of General Internal Medicine 2001;16:606-613. [PubMed: 11556941]

Kumar R, Woo MA, Birrer BVX, Macey PM, Fonarow GC, Hamilton MA, et al. Mammillary bodies and fornix fibers are injured in heart failure. Neurobiology of Disease 2009;33:236-242. [PubMed: 19022386]

Lloyd-Jones D, Adams R, Carnethon M, DeSimone G, Ferguson TB, Flegal K, et al. Heart disease and stroke statistics - 2009 update: a report from the AHA Statistics Committee and Stroke Statistics Subcommittee. Circulation 2009;119:e21-e181. 2009. [PubMed: 19075105]

National Heart, Lung, and Blood Institute. The Seventh Report of the Joint National Committee on Prevention, Detection, Evaluation, and Treatment of High Blood Pressure. May. 2003 NIH Publication no. 03-5233http://www.nhlbi.nih.gov/guidelines/hypertension/index.htm (Accessed 06-17-03)

National Institute on Aging. Growing older in America: the Health and Retirement Study. U.S. Department of Health and Human Services; Washington DC: 2007. NIH Publication No. 07-5757

New York Heart Association Criteria Committee. Disease of the Heart and Blood Vessels: Nomenclature and Criteria for Diagnosis. 6th ed.. Little, Brown \& Co; Boston, MA: 1964.

Perkins AJ, Kroenke K, Unutzer J, Katon W, Williams JW Jr. Hope C, et al. Common comorbidity scales were similar in their ability to predict health care costs and mortality. Journal of Clinical Epidemiology 2004;57:1040-1048. [PubMed: 15528055]

Peterson RC, Smith GE, Ivnik RJ, Tangalos EG, Schaid DJ, Thibodeau SN, et al. Apolipoprotein E status as a predictor of the development of Alzheimer's disease in memory-impaired individuals. JAMA 1995;273:1274-1278. [PubMed: 7646655]

Pressler SJ. Cognitive functioning and chronic heart failure: a review of the literature (2002-July 2007). Journal of Cardiovascular Nursing 2008;23:239-249. [PubMed: 18437066] 
Pullicino PM, Wadley YG, McClure LY, Safford MM, Lazar RM, Klapholz M, et al. Factors contributing to global cognitive impairment in heart failure: results from a population-based cohort. Journal of Cardiac Failure 2008;14:290-295. [PubMed: 18474341]

Putzke JD, Williams MA, Millsaps CL, Azrin RL, LaMarche JA, Bourge RC, et al. Heart transplant candidates: a neuropsychological descriptive database. Journal of Clinical Psychology in Medical Settings 1997;4:343-355.

Rains, GD. Principles of Human Neuropsychology. McGraw-Hill; Boston, MA: 2002.

Reitan, RM. Trail Making Test. Reitan Neuropsychological Laboratory; Tucson, AZ: 1992.

Rutledge T, Reis VA, Linke SE, Greenberg BH, Mills PJ. Depression in heart failure: a meta-analytic review of prevalence, intervention effects, and association with clinical outcomes. Journal of the American College of Cardiology 2006;48:1527-1537. [PubMed: 17045884]

Shumaker SA, Legault C, Rapp SR, Thal L, Wallace RB, Ockene JK, et al. Estrogen plus progestin and the incidence of dementia and mild cognitive impairment in postmenopausal women. Journal of the American Medical Association 2003;289:2651-2662. [PubMed: 12771112]

Siachos T, Vanbakel A, Feldman DS, Uber W, Simpson KN, Pereira NL. Silent strokes in patients with heart failure. Journal of Cardiac Failure 2005;11:485-489. [PubMed: 16198241]

The Psychological Corporation. Wechsler ${ }^{\mathrm{R}}$ Test of Adult Reading Manual. The Psychological Corporation; San Antonio: 2001.

Vogels RLC, Oosterman JM, van Harten B, Scheltens P, van der Flier WM, Schroeder-Tanka JM, et al. Profile of cognitive impairment in heart failure. Journal of the American Geriatrics Society 2007;55:1764-1770. [PubMed: 17727641]

Vogels RL, Scheltens P, Schroeder-Tanka JM, Weinstein HC. Cognitive impairment in heart failure: a systematic review of the literature. European Journal of Heart Failure 2007;9:440-449. [PubMed: 17174152]

Waldstein SR. The relation of hypertension to cognitive function. Current Directions in Psychological Science 2003;12:9-12.

Wechsler, D. Administration and Scoring Manual. 3rd ed.. The Psychological Corporation; USA: 1997. Wechsler Adult Intelligence Scale.

Welsh KA, Butters N, Mohs RC, Beekly D, Edland S, Fillenbaum W, et al. The Consortium to Establish a Registry for Alzheimer's Disease (CERAD). Part V. A normative study of the neuropsychological battery. Neurology 1994;44:609-614. [PubMed: 8164812]

Woo MA, Kumar R, Macey PM, Fonarow GC, Harper RM. Brain injury in autonomic, emotional, and cognitive regulatory areas in patients with heart failure. Journal of Cardiac Failure 2009;15:214223. [PubMed: 19327623]

Woo MA, Macey PM, Fonarow GC, Hamilton MA, Harper RM. Regional brain gray matter loss in heart failure. Journal of Applied Physiology 2003;95:677-684. [PubMed: 12716866]

Zipes, DP.; Libby, P.; Bonow, RO.; Braunwald, E. Braunwald's heart disease: a textbook of cardiovascular medicine. 7th ed.. Elsevier Saunders; Philadelphia: 2005.

Zuccala G, Pedone C, Cesari M, Onder G, Pahor M, Marzetti E, et al. The effects of cognitive impairment on mortality among hospitalized patients with heart failure. American Journal of Medicine 2003;115:97-103. [PubMed: 12893394] 
Table 1

\section{Cognitive Domain, Neuropsychological Tests, and Tests Descriptions}

\begin{tabular}{|c|c|c|}
\hline Cognitive domain & Neuropsychological tests ${ }^{a}$ & Brief description \\
\hline Global cognitive function & Mini-mental State Examination $b$ & $\begin{array}{l}\text { Participants complete a } 30 \text {-point screening questionnaire to } \\
\text { evaluate general mental status. Higher scores indicate better } \\
\text { mental status. }\end{array}$ \\
\hline Premorbid intellect & Wechsler Test of Adult Reading $c$ & $\begin{array}{l}\text { Participants asked to pronounce a list of phonologically } \\
\text { irregular words aloud. Measure of analysis was a standard score } \\
\text { with mean }=100 \text { and standard deviation }=15 \text {. }\end{array}$ \\
\hline Language & Boston Naming Test $d$ & $\begin{array}{l}\text { Participants name line drawings of common objects. Possible raw } \\
\text { scores range from } 0 \text { to } 60 \text {, with higher scores indicating } \\
\text { better ability. }\end{array}$ \\
\hline Working memory & Digit Span ${ }^{e}$ & $\begin{array}{l}\text { Participants repeat series of numbers forward and backward. } \\
\text { The raw forward and backward spans were analyzed separately } \\
\text { (possible forward score } 0 \text { to } 16 \text {; possible backward score } 0 \text { to } 14 \text { ) } \\
\text { and the total score was transformed to a scaled score (mean =10 } \\
\text { and standard deviation }=3 \text { ) as derived from the WAIS-3. }\end{array}$ \\
\hline Verbal memory & Hopkins Verbal Learning Test $f$ & $\begin{array}{l}\text { Participants asked to learn orally presented } 12 \text {-word list over } \\
\text { three rehearsal trials, with recall after each list presentation. After } \\
\text { a } 20 \text {-minute delay, they are asked to recall as many of the } 12 \text { words } \\
\text { as they can. The variables analyzed were total learning over the } \\
\text { three trials (possible score } 0 \text { to } 36 \text { for summed recall of trials } 1 \\
\text { through } 3 \text { ) and delayed recall (possible score } 0 \text { to } 12 \text { ). Higher } \\
\text { scores indicate better performance. }\end{array}$ \\
\hline Visuospatial ability & $\begin{array}{l}\text { Figure Copy and Figure } \\
\text { Memory recall } g\end{array}$ & $\begin{array}{l}\text { Participants are shown five figures that increase in complexity } \\
\text { (circle, diamond, intersection, rectangle, and cube) and are asked } \\
\text { to draw a copy of each figure. After a } 20 \text {-minute delay, they are } \\
\text { asked to recall and draw the figures again. The variables analyzed } \\
\text { were total (possible scores range from } 0 \text { to } 11 \text { ) and recall (possible } \\
\text { scores range from } 0 \text { to } 14 \text { ) with higher scores indicating better } \\
\text { performance. }\end{array}$ \\
\hline \multirow[t]{2}{*}{ Psychomotor speed } & Digit Symbol $^{e}$ & $\begin{array}{l}\text { Participants required to match a series of numbers with symbols } \\
\text { using a key that shows the unique pairing of the numbers with the } \\
\text { symbols. The measure of analysis was the scaled score derived } \\
\text { from the WAIS- } 3 \text { standardization sample (mean }=10 \text {; standard } \\
\text { deviation }=3 \text { ). }\end{array}$ \\
\hline & Trail Making Test, Part $\mathrm{A}^{h}$ & $\begin{array}{l}\text { Participants use a pencil to draw a line that connects numbered } \\
\text { circles arrayed on a page as fast as possible. The measure of } \\
\text { analysis was the number of seconds to complete the task, with } \\
\text { higher scores (longer time) indicating slower psychomotor speed. }\end{array}$ \\
\hline \multirow[t]{2}{*}{ Executive function } & Trail Making Test, Part $\mathrm{B}^{h}$ & $\begin{array}{l}\text { Participants use a pencil to connect numbered and lettered circles } \\
\text { sequentially as fast as possible, but in alternate alpha-numeric } \\
\text { order (i.e., number-letter-number-letter). The measure of analysis } \\
\text { is the number of seconds to complete the task, with higher scores } \\
\text { (longer time) indicating poorer shifting between numbers } \\
\text { and letters. }\end{array}$ \\
\hline & Controlled Oral Word Association ${ }^{i}$ & $\begin{array}{l}\text { Participants given a letter of the alphabet and asked to name as } \\
\text { many words as possible that begin with that letter. The test } \\
\text { measures the speed with which participants can search and } \\
\text { retrieve a word from their mental lexicon using a phonemic stem. } \\
\text { The measure of analysis was the number of words produced in } 90 \\
\text { seconds for each of the letters C, F, and L, with higher scores } \\
\text { indicating better performance. }\end{array}$ \\
\hline
\end{tabular}

${ }^{a}$ Although the tests were used to sample a specific domain, each test actually samples multiple domains.

${ }^{b}$ Folstein, Folstein, \& McHugh, 1975

$c_{\text {The Psychological Corporation. Wechsler }}{ }^{R}$ Test of Adult Reading Manual. San Antonio: The Psychological Corporation; 2001.

$d_{\text {Kaplan, Goodglass, \& Weintraub, } 2001}$

$e_{\text {Wechsler, } 1997}$

Nurs Res. Author manuscript; available in PMC 2011 March 1. 


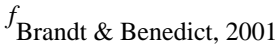

${ }^{g}$ Welsh et al., 1994

$h_{\text {Reitan, } 1992}$

${ }^{i}$ Benton \& Hamsher, 1978 


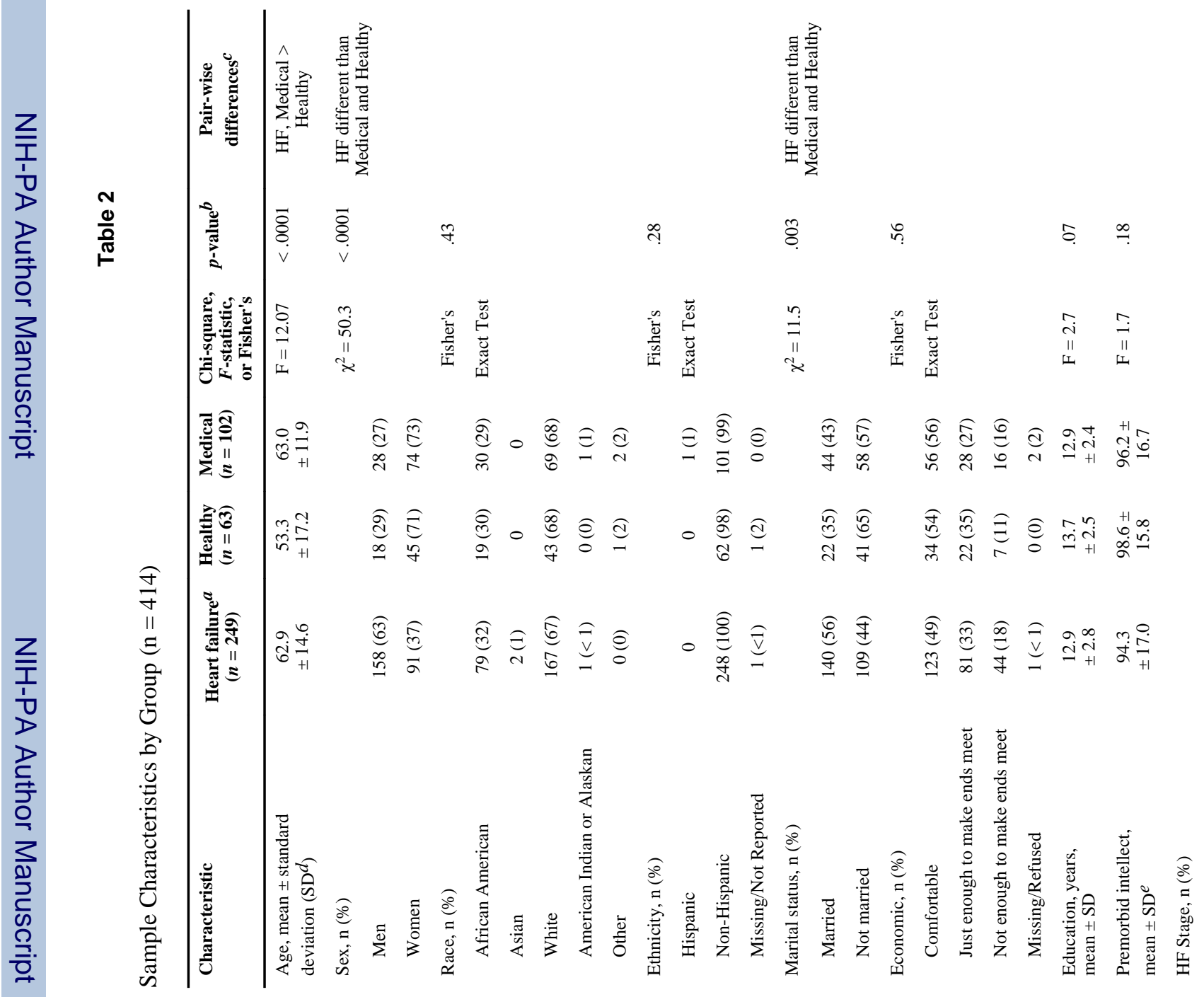
Nurs Res. Author manuscript; available in PMC 2011 March 1. 


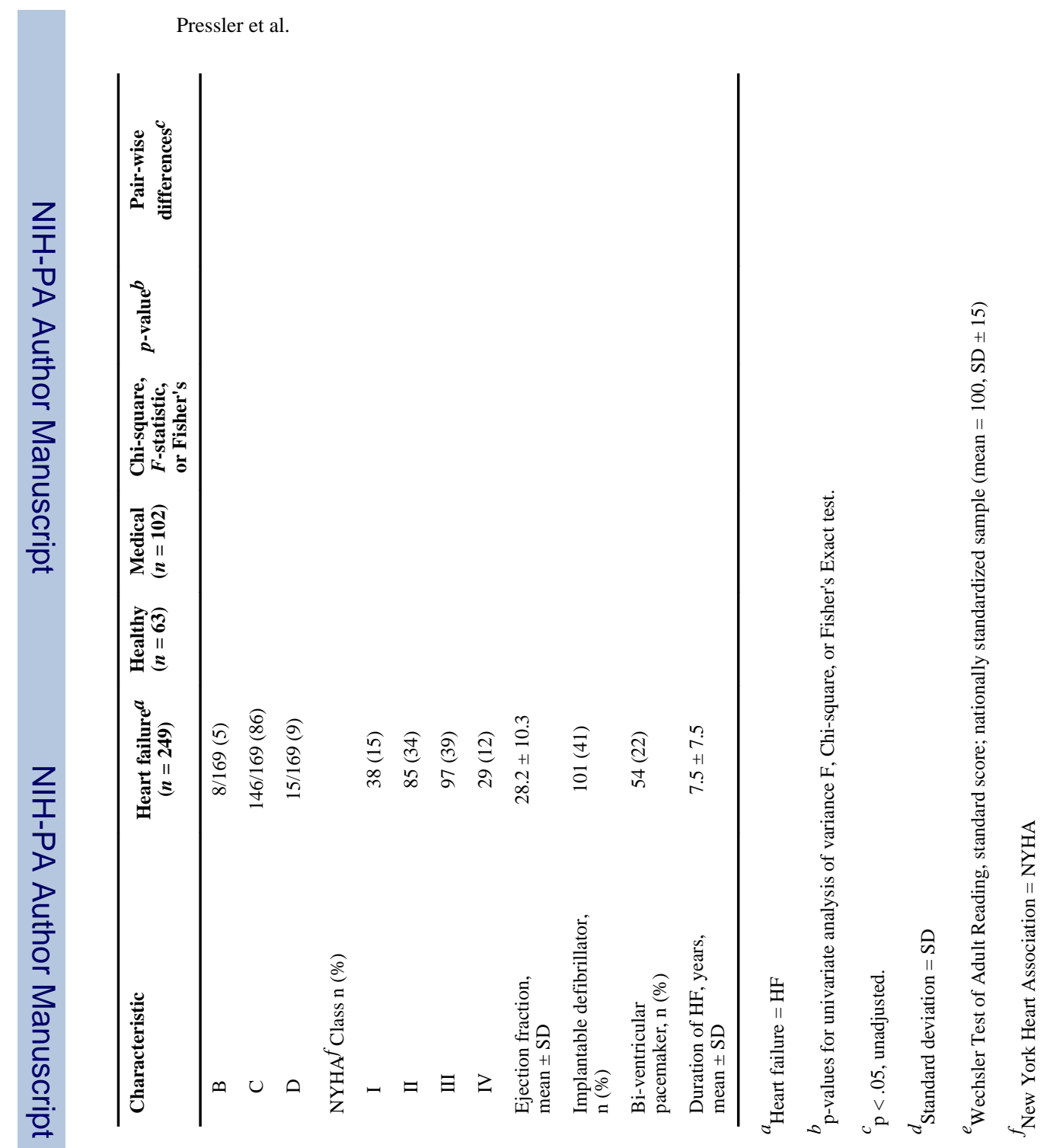

Page 16 


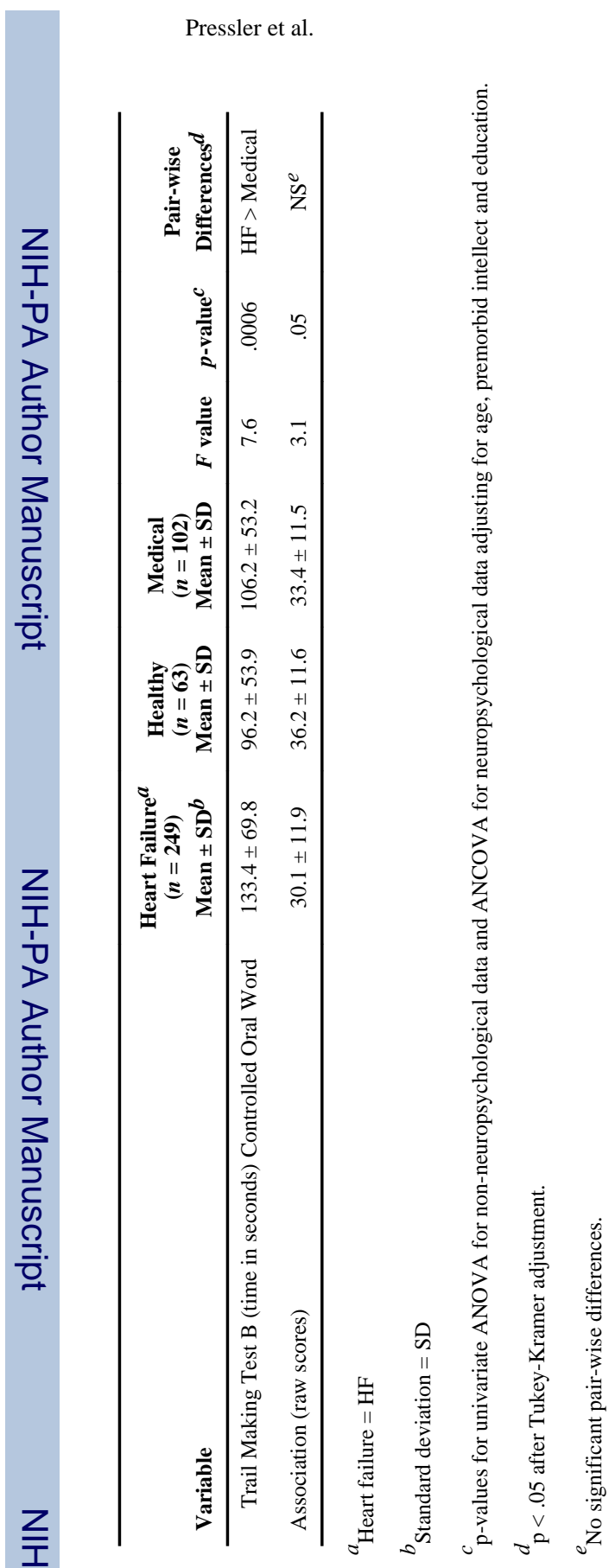

Nurs Res. Author manuscript; available in PMC 2011 March 1. 


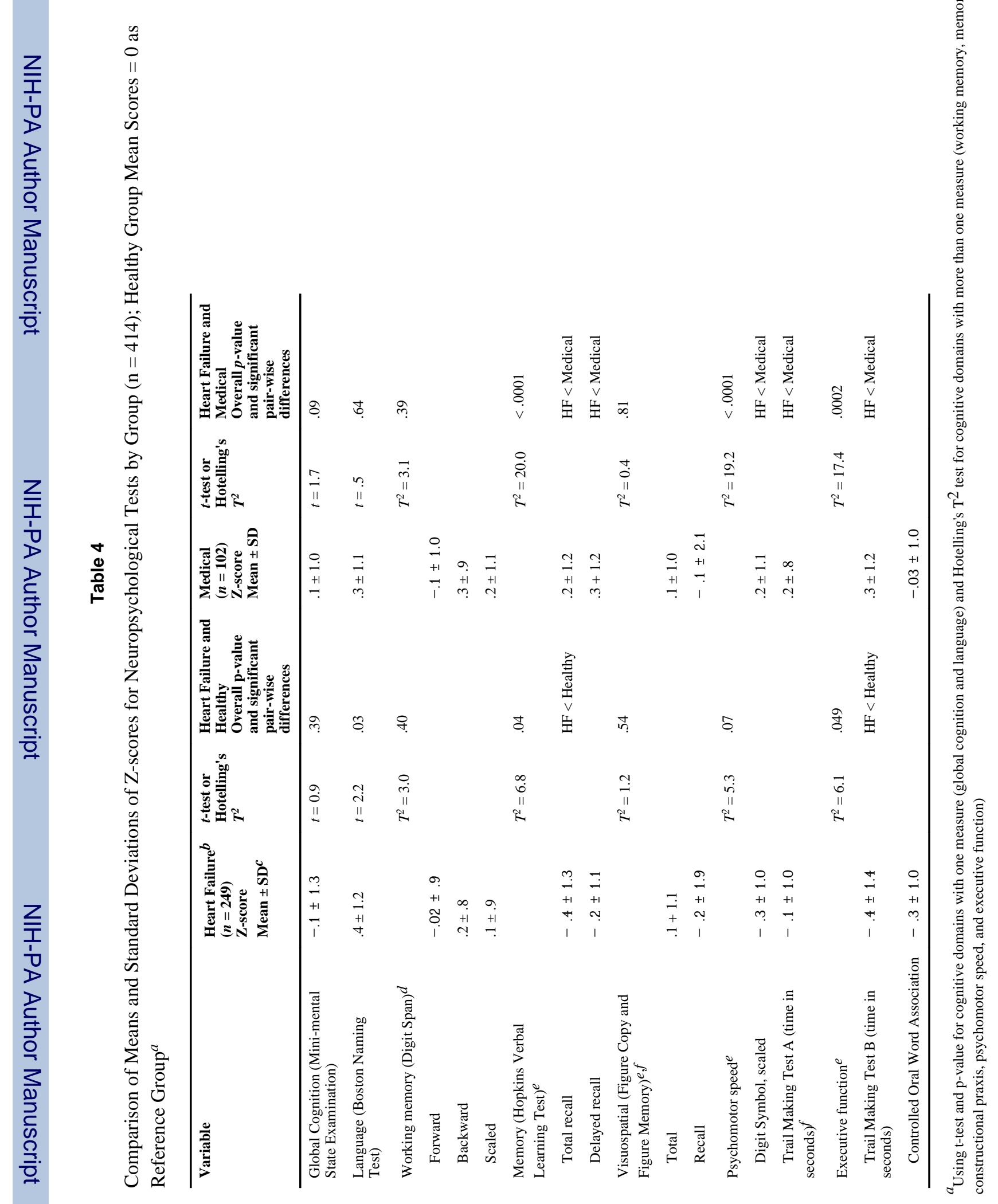




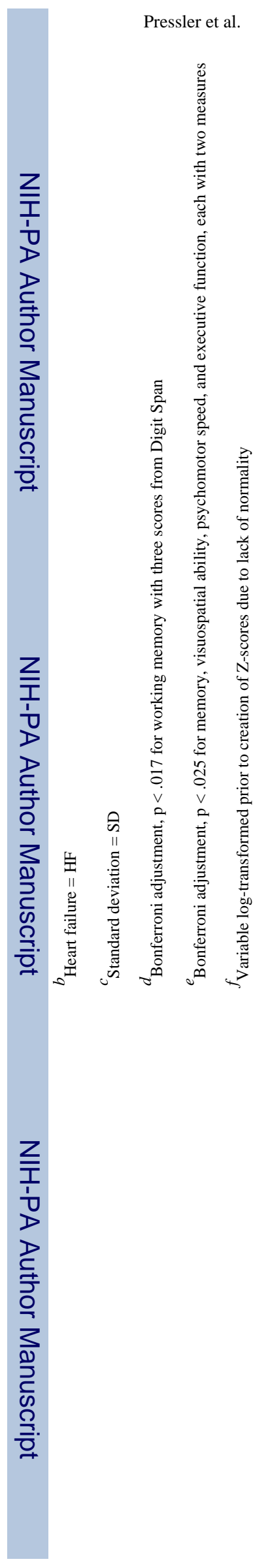

Page 20

Nurs Res. Author manuscript; available in PMC 2011 March 1. 
